\title{
Disrupting the Perception of Effort with Continuous Theta Burst Stimulation
}

\author{
두revor T.-J. Chong \\ Department of Cognitive Science, Macquarie University, Sydney, New South Wales 2109, Australia \\ Review of Zénon et al.
}

A substantial challenge facing empirical investigations of effort perception is the necessity of approaching an inherently private phenomenological experience with scientific rigor. In the $19^{\text {th }}$ century, Bell (1826) and Sherrington (1900) proposed that the sensation of muscular forces originates from peripheral receptors, while Helmholtz (1867) posited that the perception of effort arises from internal signals generated by the central motor command. Although the question of how effort is perceived remains controversial, a current view, echoing that of Helmholtz, is that it arises primarily from efferent activity within the motor system. According to this model, when the brain initiates a motor command, it issues a parallel neural representation to predict the sensory consequences of that action (the corollary discharge or efference copy), which can then be used as an indicator of how subjectively effortful that action is (Carson et al., 2002). The centrality of the efference copy in effort perception is consistent with data showing that deafferented patients or those with suppressed sensory afferents can never-

Received July 9, 2015; revised Aug. 19, 2015; accepted Aug. 22, 2015.

T.T.-J.C. is supported by a National Health and Medical Research Council (NH\&MRC) of Australia Neil Hamilton Fairley Early Career Fellowship.

The author declares no competing financial interests.

Correspondence should be addressed to Dr Trevor T.-J. Chong, Department of Cognitive Science, Macquarie University, Sydney, NSW 2109, Australia. E-mail: trevor.chong@mq.edu.au.

DOI:10.1523/JNEUROSCI.2624-15.2015

Copyright $\odot 2015$ the authors $\quad 0270-6474 / 15 / 3513269-03 \$ 15.00 / 0$ theless perceive actions as effortful (Marcora, 2009).

Based on these models, Zénon and colleagues (2015) attempted to modulate effort perception by using continuous theta burst stimulation (cTBS) to disrupt the supplementary motor area (SMA) - an area whose activity correlates with grip force intensity, and is implicated as a source of the efference copy. Each trial of their task required participants to squeeze a hand-held dynamometer at one of four levels of force in return for various (randomly allocated) rewards, while receiving visual feedback of their contraction. At the conclusion of each squeeze, participants either subjectively rated their perceived exertion, or indicated whether they were willing to replicate their contraction to double their reward. Higher replication rates were taken to imply lower levels of perceived effort. If the participants chose to replicate the contraction, they were then asked to squeeze the dynamometer a second time in the absence of visual feedback, and were rewarded according to their force-matching accuracy. A lower force output on the replication compared with the reference trial was taken to imply lower levels of perceived effort in the reference contraction. In addition, the authors measured pupillary diameter as a physiological indicator of arousal, and computed the effect of force in each trial on the force generated in the first contraction of the subsequent trial. According to the authors, the rationale for the latter measure was that each contraction leads to an expectation that the following trial will require a contraction of similar force; thus, a contraction perceived as more effortful should lead to the generation of greater force on the next trial. The effect of SMA stimulation was compared with the effects of stimulation of the primary motor cortex (M1) and a control site over the precuneus. The precuneus itself has been proposed to be involved in a diversity of functions, including imagery and episodic memory (Cavanna and Trimble, 2006). Each site was tested on a separate day and, on each day, participants were tested in two sessions-before and after cTBS.

The authors found consistent effects of cTBS over the SMA relative to the precuneus on all five dependent variables. Specifically, SMA stimulation relative to the precuneus resulted in lower subjective effort ratings, less physiological arousal, lower effort replication intensities, higher rates of replication as a function of reward, and an attenuated effect of force in each trial on force output on the subsequent trial (albeit only for the lowest two effort levels). In addition, structural equation modeling revealed that disruption of SMA, but not $\mathrm{M} 1$, led to a decrease in effort perception, which was modeled as a latent variable dependent on the five aforementioned measures. Importantly, although maximum voluntary con- 
tractions decreased over the course of the experiment due to fatigue, cTBS over the three sites did not differentially alter the motor threshold.

One of the main strengths of this study was its use of multiple variables to verify the effects of cTBS on effort. However, these measures also highlight the challenges of quantifying effort perception empirically. As the authors themselves acknowledge, interpreting some of their measures may not be straightforward. For example, several findings could conceivably be explained without reference to subjective effort, but simply as effects on motor precision. Consider force-matching accuracy, or the influence of one contraction on the force generated in the subsequent trial. Both measures do indeed rely on the perceived effort of the previous contraction, but they are also contingent on motor memory, motor imagery, and the ability to produce a contraction in the presence or absence of visual feedback, all of which could be differentially affected by cTBS over the target or control sites. Thus, one could very reasonably argue that the authors' findings with regards to force-matching, or the effect of one contraction on the subsequent trial, were due to mismatches between the efference copy and reafferent input leading to impaired motor precisionhowever, such an explanation need not invoke any conceptual link to subjective effort whatsoever. Nevertheless, the value of this study stems from the convergence of measures to bolster its case, because taken together, the data provide evidence in the form of subjective selfreport measures, physiological measures of arousal, and objective measures of motor precision, to collectively suggest that effort perception can be modulated by SMA relative to control site stimulation.

Interestingly, although SMA stimulation showed significant effects relative to precuneus stimulation, there seemed to be no effect of either SMA or M1 stimulation in isolation on several objective measures (namely, effort replication intensity, pupil size, and perhaps force prediction, although direct statistical comparisons were not made between prestimulation and poststimulation blocks) (Zénon et al., 2015, their Fig. 3). Indeed, subjective effort ratings were the only measure that was lower after SMA or M1 stimulation than during the prestimulation period, and these lower effort ratings might be explicable simply on the basis that participants were in fact exerting less force in poststimulation blocks due to fatigue. When considering the results as a function of individual stimulation sites, the most striking effects of cTBS appeared to be at the precuneus. One interesting speculation therefore is that the precuneus control site might have contributed unexpectedly to the results in this study. The precuneus has strong connections with motor areas, including the SMA, dorsal premotor cortex, and parietal areas implicated in the efference copy; functionally, there is evidence to suggest that it is involved in episodic memory and motor imagery (Cavanna and Trimble, 2006). It is therefore possible that several measures might have been affected by precuneal stimulation (e.g., replication rate, effort replication intensity). The possibility that the precuneus was not an entirely neutral site could explain why there were no significant differences between $\mathrm{M} 1$ and precuneus cTBS on most measures, which seems at odds with recent claims that M1 stimulation modulates effort perception in a force-matching task (Takarada et al., 2014). Future studies should clarify these discrepancies by incorporating a sham condition and/or including a different control site. For now, the results of the present study suggest that SMA disruption does not uniformly decrease effort perception in absolute terms, but only relative to precuneus cTBS.

It will also be important for future work to determine the mechanism underlying reduced effort perception. Although current theories of effort perception downplay the role of afferent inputs, several authors have attempted to re-emphasize their importance (Luu et al., 2011; Scotland et al., 2014). Indeed, depending on task instructions, participants can shift their judgments of effort based on either the efferent command or afferent input about muscular tension. The contribution of afferent inputs to the perception of effort would not be surprising-after all, a forward model of motor control predicts that the efference copy should be projected to somatosensory cortex, and a comparator should convey the error in that efferent prediction with reafferent input (Wolpert and Ghahramani, 2000). Thus, although Zénon and colleagues (2015) argue that their modulation of effort perception was caused by disrupting some form of efference copy, a nonmutually exclusive possibility is that afferent inputs indirectly contribute to effort perception by calibrating or modulating the activity of the comparator.
Future studies should examine the relative contributions to effort perception of regions putatively involved in generating the efference copy itself (e.g., the SMA) versus those thought to compute the internal models that predict and update the sensory consequences of that action (e.g., the cerebellum or posterior parietal cortex).

The question of whether effort perception can be modulated is a topical one, given the recent surge of interest in characterizing effort computationally and with fMRI (Skvortsova et al., 2014). The finding that it may be possible to attenuate the perception of physical effort by disrupting the SMA opens several avenues for further enquiry. For example, effort perception in humans extends beyond the physical domain to the cognitive one as well. Given the intrinsic differences between cognitive and physical effort, a challenge for future research would be to identify a putative analog to the SMA for cognitive effort perception and test its causal role by using neurodisruptive techniques such as those used here. A further question is how SMA cTBS disrupts the integration of effort and reward. The finding that replication rates increased as a function of reward only following SMA stimulation suggests some degree of value integration between effort and reward at that site (cf. Croxson et al., 2009). However, rewards in the present study were randomly allocated, and future studies would be apt to dissect this interaction by systematically manipulating reward as a variable. More broadly, although the precise theoretical underpinnings of effort perception remain to be dissected, Zénon and colleagues (2015) provide important data for the potential development of useful therapeutic strategies that can modulate effort perception in patient populations, such as those who suffer from debilitating symptoms of fatigue.

\section{References}

Bell C (1826) On the nervous circle which connects the voluntary muscles with the brain. Philos Trans R Soc Lond 116:163-173.

Carson RG, Riek S, Shahbazpour N (2002) Central and peripheral mediation of human force sensation following eccentric or concentric contractions. J Physiol 539:913-925. CrossRef Medline

Cavanna AE, Trimble MR (2006) The precuneus: a review of its functional anatomy and behavioural correlates. Brain 129:564-583. CrossRef Medline

Croxson PL, Walton ME, O'Reilly JX, Behrens TE, Rushworth MF (2009) Effort-based cost-benefit 
valuation and the human brain. J Neurosci 29: 4531-4541. CrossRef Medline

Helmholtz Hv (1867) Handbuch der Physiologischen Optik B. Leipzig: Voss.

Luu BL, Day BL, Cole JD, Fitzpatrick RC (2011) The fusimotor and reafferent origin of the sense of force and weight. J Physiol 589:31353147. CrossRef Medline

Marcora S (2009) Perception of effort during exercise is independent of afferent feedback from skeletal muscles, heart, and lungs. J Appl Physiol 106:2060-2062. CrossRef Medline
Scotland S, Adamo DE, Martin BJ (2014) Sense of effort revisited: relative contributions of sensory feedback and efference copy. Neurosci Lett 561:208-212. CrossRef Medline

Sherrington C (1900) The muscular sense. In: Textbook of physiology (Shafer E, ed.), pp 1002-1025. Edinburgh: Pentland.

Skvortsova V, Palminteri S, Pessiglione M (2014) Learning to minimize efforts versus maximizing rewards: computational principles and neural correlates. J Neurosci 34:15621-15630. CrossRef Medline
Takarada Y, Mima T, Abe M, Nakatsuka M, Taira M (2014) Inhibition of the primary motor cortex can alter one's "sense of effort": effects of low-frequency rTMS. Neurosci Res 89:5460. CrossRef Medline

Wolpert DM, Ghahramani Z (2000) Computational principles of movement neuroscience. Nat Neurosci 3:1212-1217. CrossRef Medline

Zénon A, Sidibé M, Olivier E (2015) Disrupting the supplementary motor area makes physical effort appear less effortful. J Neurosci 35: 8737-8744. CrossRef Medline 